\title{
Production of an anti-TM4SF5 monoclonal antibody and its application in the detection of TM4SF5 as a possible marker of a poor prognosis in colorectal cancer
}

\author{
BYOUNG KWON PARK ${ }^{1 *}$, JAE-YOUNG PARK ${ }^{2 *}$, TE HA KIM $^{3}$, DONGBUM KIM ${ }^{1}$, GUANG WU $^{1}$, \\ AVISHEKH GAUTAM ${ }^{3}$, SONY MAHARJAN ${ }^{1}$, SU IN LEE ${ }^{1}$, YOUNGHEE LEE ${ }^{4}$, \\ HYUNG-JOO KWON ${ }^{1,3}$ and KYUNG CHAN CHOI ${ }^{1,2}$
}

\author{
${ }^{1}$ Center for Medical Science Research, College of Medicine, Hallym University, Chuncheon 24252; \\ ${ }^{2}$ Department of Pathology, Hallym University Sacred Heart Hospital, Chuncheon 24253; ${ }^{3}$ Department of Microbiology, \\ College of Medicine, Hallym University, Chuncheon $24252 ;{ }^{4}$ Department of Biochemistry, \\ College of Natural Sciences, Chungbuk National University, Cheongju 28644, Republic of Korea
}

Received January 8, 2018; Accepted April 20, 2018

DOI: $10.3892 /$ ijo.2018.4385

\begin{abstract}
The cell surface transmembrane 4 superfamily member 5 protein (TM4SF5) has been implicated in various human cancers. Immunization with a peptide vaccine targeting human TM4SF5 has been shown to exert prophylactic and therapeutic effects against the development of hepatocellular carcinoma and colon cancer in mouse models. In this study, we developed a novel monoclonal antibody (mEC2-CF) targeting a cyclic epitope of TM4SF5 and evaluated its reactivity to TM4SF5 in colorectal cancer (CRC) cells and cancer tissues. The isotype of mEC2-CF was IgG2a and the antibody specifically recognized the cyclic peptide, based on ELISA. The antibody recognized recombinant TM4SF5 overexpressed in $293 \mathrm{~F}$ cells, irrespective of $\mathrm{N}$-glycosidase $\mathrm{F}$ treatment. The
\end{abstract}

Correspondence to: Dr Hyung-Joo Kwon, Department of Microbiology, College of Medicine, Hallym University, 1 Hallymdaehak-gil, Chuncheon 24252, Republic of Korea

E-mail:hjookwon@hallym.ac.kr

Dr Kyung Chan Choi, Department of Pathology, Hallym University Sacred Heart Hospital, 77 Sakju-ro, Chuncheon 24253, Republic of Korea

E-mail: kcchoi@hallym.or.kr

${ }^{*}$ Contributed equally

Abbreviations: CRC, colorectal cancer; EC1, short extracellular loop 1; EC2, long extracellular loop 2; ELISA, enzyme-linked immunosorbent assay; $\mathrm{HCC}$, hepatocellular carcinoma; mEC2-CF, anti-hTM4SF5EC2-CF monoclonal antibody; PNGase, N-glycosidase; TM4SF5, transmembrane 4 superfamily member 5 protein

Key words: transmembrane 4 superfamily member 5 protein, monoclonal antibody, immunohistochemistry, prognosis, colorectal cancer antibody was internalized into the cytosol after binding to the surface of TM4SF5-expressing CRC cells, suggesting that this antibody may be useful in therapeutics. In addition, we evaluated TM4SF5 expression in the tissues of patients with CRC patients to determine its prognostic significance. TM4SF5 expression was assessed by immunohistochemistry using mEC2-CF and tissue microarray blocks of 204 primary CRC samples. The overall rate of TM4SF5 overexpression in the samples (immunohistochemical score $>4$ ) was $27.0 \%$ (55 of 204). The increased expression of TM4SF5 was significantly associated with a shorter survival rate $(\mathrm{P}=0.0014)$ and a worse disease-free survival $(\mathrm{P}=0.0483)$ of patients with CRC. No association was observed between TM4SF5 expression and clinicopathological characteristics, apart from tumor depth of invasion $(\mathrm{P}=0.027)$. These results suggest that our novel antibody can be used to detect endogenous and recombinant TM4SF5, and that TM4SF5 may be a possible marker for the poor prognosis of patients with CRC.

\section{Introduction}

Colorectal cancer (CRC) is associated with a high mortality rate worldwide (1). It is the second and third most common cause of cancer-related mortality for males and females, respectively (2). Despite advances in the diagnosis of CRC and treatment with surgery, chemotherapy and radiotherapy, numerous patients still succumb to the disease (3). Therefore, additional studies focusing on novel methods for the diagnosis and treatment of the disease are required.

Transmembrane 4 superfamily member 5 protein (TM4SF5) is eminently expressed in colon, liver, esophageal and pancreatic cancers (4-10). TM4SF5 is composed of four transmembrane regions, two cytoplasmic tails ( $\mathrm{N}$ - and $\mathrm{C}$-terminal), and two extracellular loops [short extracellular loop 1 (EC1) and long extracellular loop 2 (EC2)] (11). TM4SF5 forms an enormous tetraspanin-enriched domain by interacting with other TM4SFs and integrins to accomplish various functional roles, including roles in cell growth, migration, tumor progression, and 
metastasis $(12,13)$. TM4SF5 induces epithelial-mesenchymal transition by increasing $\mathrm{p} 27^{\mathrm{kip} 1}$ expression and stabilizing the cytosolic form of $\mathrm{p} 27^{\mathrm{kip} 1}$, resulting in abnormal cell growth, the loss of contact inhibition and tumor progression (5). TM4SF5 activates focal adhesion kinase (FAK) and c-Src, which in turn increase cell adhesion, migration and lung metastasis (14). Furthermore, epidermal growth factor receptor and integrin $\alpha 5$ complexes with TM4SF5 mediate cell migration and invasion (15). EC2 of TM4SF5 contains N-glycosylation sites that mediate the cross-talk with integrins, thereby affecting cellular migration and invasion (16). Blocking the EC2 domain with a synthetic anti-TM4SF5 compound, 4'-(p-toluenesulfonylam ido)-4-hydroxychalcone (TSAHC), has been shown to inhibit TM4SF5-mediated tumorigenesis (6). Therefore, TM4SF5 is considered a major molecular target for anticancer therapy and EC2 is regarded as a key region for the inhibition of TM4SF5 activity.

We previously developed a prophylactic and therapeutic vaccine for colon and hepatocellular carcinoma (HCC) using an EC2 domain TM4SF5 peptide epitope (hTM4SF5R2-3, ${ }^{138}$ NRTLWDRCEAPPRV ${ }^{151}$ ) in combination with our own adjuvant formulation complex [Lipoplex(O)] (7-9). We then generated a mouse monoclonal antibody against the hTM4SF5R2-3 epitope, which displayed therapeutic effects in colon and HCC mouse tumor models $(17,18)$. Moreover, using a novel type of cyclic peptide epitope targeting the EC2 domain (hTM4SF5EC2-C, ${ }^{131}$ TACAYLLNRTLWDRSEAPPRVVPWNC $T^{157}$ ), we also produced a humanized anti-TM4SF5 antibody that decreased the generation and growth of lung metastasis in a mouse colon cancer model (19).

In this study, we successfully produced a monoclonal antibody targeting hTM4SF5 using another cyclic peptide epitope mimicking the natural structure of the EC2 domain (hTM4SF5EC2-CF, ${ }^{116}$ PRCLMNGEWGYHFED TAGAYLLNRTLWDRCEAPPRVVP(53). We also examined the utility of this monoclonal antibody for TM4SF5 recognition in cancer tissues and then employed this antibody in evaluation of TM4SF5 as a prognostic marker for patients with CRC.

\section{Materials and methods}

Cell culture. The $293 \mathrm{~F}$ cell line was purchased from the Thermo Fisher Scientific (Waltham, MA, USA) and maintained in Freestyle 293 Expression Medium (Thermo Fisher Scientific). The human colon cancer cell lines, HT-29, LoVo and HCT-116, were purchased from the Korean Cell Line Bank (Seoul, Korea) and maintained in RPMI-1640 medium (Thermo Fisher Scientific). All media were supplemented with $10 \%$ fetal bovine serum (FBS; Thermo Fisher Scientific), $25 \mathrm{mM}$ HEPES, $100 \mathrm{U} / \mathrm{ml}$ penicillin and $100 \mu \mathrm{g} / \mathrm{ml}$ streptomycin. All cell lines were incubated at $37^{\circ} \mathrm{C}$ in an atmosphere of $95 \%$ air and $5 \% \mathrm{CO}_{2}$.

Synthesis of TM4SF5 B-cell-epitope peptides. To select B-cell-epitope peptides for hTM4SF5-specific antibody production, we analyzed the epitope probability, surface accessibility and antigenicity index (http://tools.iedb.org/bcell) of the hTM4SF5 protein. We selected several epitope sequences from the EC2 domain of TM4SF5 (Fig. 1A and B). We synthesized each peptide, including cyclic peptides, with an automated peptide synthesizer (Peptron III-R24; Peptron, Daejeon, Korea). The peptides were purified by reversed-phase HPLC (Prominence HPLC; Shimadzu Corp., Tokyo, Japan) to a $>90 \%$ purity.

Mice. Four-week-old female BALB/c $\left(\mathrm{H}-2^{b}\right)$ mice $(\mathrm{n}=11$; weight, 19-20 g) were purchased from Nara-Biotec (Seoul, Korea). Our animal experiments were approved by the Institutional Animal Care and Use Committee of Hallym University (Permit no. Hallym 2015-55, Hallym 2015-56). The mice were maintained under specific pathogen-free conditions in a controlled environment $\left(20-25^{\circ} \mathrm{C}, 40-45 \%\right.$ humidity, 12-h light/dark cycle; ad libitum access to food and water). The mice were euthanized by $\mathrm{CO}_{2}$ inhalation $\left(\mathrm{CO}_{2}\right.$ inhalation was performed with $100 \% \mathrm{CO}_{2}$ at a fill rate of $10-30 \%$ of the chamber volume per min) when the mice lost and gained $20 \%$ of adult body weight, exhibited evidence of debilitation, pain or distress, such as a hunched posture, rough haircoat, decreased food consumption, emaciation, inactivity, difficulty ambulating, respiratory problems and solid tumor growth. After the experiments were completed, the mice were sacrificed by $\mathrm{CO}_{2}$ inhalation (as mentioned above) and all efforts were made to minimize suffering.

Immunization. Epitope peptide-CpG-DNA-liposome complex [Lipoplex(O)] was formulated as previously described $(20,21)$. Briefly, the liposome complexes consisting of hTM4SF5EC2-CF cyclic peptide and CpG-DNA [MB-ODN 4531(O)] co-encapsulated with phosphatidyl- $\beta$-oleoyl- $\gamma$-palmitoyl ethanolamine (DOPE):cholesterol hemisuccinate (CHEMS) were prepared. The MB-ODN 4531(O) encapsulated in DOPE:CHEMS (at a 1:1 ratio) complex as was named Lipoplex(O). The mixture of DOPE and CHEMS was prepared in ethanol at a molar ratio of 1:1, and evaporated with nitrogen gas, and then resuspended in water-soluble CpG-DNA and hTM4SF5EC2-CF cyclic peptide. Following vigorous stirring at room temperature for $30 \mathrm{~min}$, the mixture solution was adjusted the $\mathrm{pH}$ to 7.0. The mixture solution was slightly sonicated for $30 \mathrm{sec}$ with a sonicator, filtered with a $0.22 \mu \mathrm{m}$ filter, and then freeze-thawed 3 times with liquid nitrogen. The mice $(n=6$; now weighing 20-22 g) were injected intraperitoneally with $50 \mu \mathrm{g}$ of the hTM4SF5EC2-CF cyclic peptide epitope mixed with $50 \mu \mathrm{g}$ of CpG-DNA encapsulated in liposomes as previously described (20). All mice were injected on 3 occasions at 10-day intervals.

Antigen-specific Ig enzyme-linked immunosorbent assay (ELISA). Ten days after final injection of a complex of hTM4SF5EC2-CF cyclic peptide and Lipoplex(O) into the mice ( $\mathrm{n}=3$; weight, $20-25 \mathrm{~g})$, sera $(100 \mu \mathrm{l} /$ mouse $)$ were collected by retro-orbital bleeding under isoflurane (2-3\%) inhalation anesthesia with RC2-Rodent Circuit Controller (Lab Etc Inc. Store, Clayton, MO, USA) to detect peptide epitope-specific antibody production. The hTM4SF5EC2-CF peptide was coated onto 96-well immunoplates $(5 \mu \mathrm{g} /$ well; Thermo Fisher Scientific) and the plates were then blocked with phosphate-buffered saline (PBS) with $0.2 \%$ Tween-20 (PBST) containing $1 \%$ bovine serum albumin (BSA). Antibody production levels in sera were analyzed as previously described (21). 
To identify the specificity of the monoclonal antibody, various TM4SF5 peptides (Fig. 1B) were coated onto 96-well immunoplates and the plates were incubated at $4{ }^{\circ} \mathrm{C}$ overnight. The plates were blocked with PBS containing $1 \%$ BSA for $1 \mathrm{~h}$ and incubated with the mEC2-CF monoclonal antibody (serial 1:4 dilutions in PBST, beginning at $10 \mu \mathrm{g} / \mathrm{ml}$ ) for $2 \mathrm{~h}$. After the plates were washed, they were treated with horseradish peroxidase (HRP)-conjugated goat anti-mouse $\operatorname{IgG}(1: 5,000$, cat. no. 115-035-003; Jackson ImmunoResearch Laboratories, West Grove, PA, USA). The immunoreactivity was measured with a kit purchased from KPL (Gaithersburg, MD, USA) and quantified as previously described (22). The IgG isotypes were identified with HRP-conjugated goat anti-mouse IgG (each isotype) antibodies (1:500, cat. no. 5300-05; Southern Biotech, Birmingham, AL, USA). The binding affinity of the mEC2-CF monoclonal antibody was measured as previously described (14). To measure the binding affinity (EC50 value) of the mEC2-CF monoclonal antibody, 96-well immunoplates were coated with hTM4SF5EC2-CF peptide (5 $\mu \mathrm{g} / \mathrm{well})$ and then treated with PBST containing 1\% BSA. The mEC2-CF monoclonal antibody was applied to the top row of each well with serial 1:5 dilutions in PBST, starting at $1 \mu \mathrm{M}$. The mixture was incubated for $2 \mathrm{~h}$ at room temperature, and then washed with PBST. The mEC2-CF monoclonal antibody binding to hTM4SF5EC2-CF cyclic peptide was detected by incubation with the HRP-conjugated goat anti-mouse $\operatorname{IgG}(1: 5,000$; Jackson ImmunoResearch Laboratories) for $1 \mathrm{~h}$. The amounts of bound antibodies were analyzed with tetramethylbenzidine (TMB) peroxidase substrate from KPL. The absorbance was detected with the Spectra Max 250 microplate reader at $405 \mathrm{~nm}$, and the results were then analyzed with the SigmaPlot program to calculate the EC50 value.

Production of the mEC2-CF monoclonal antibody. The hybridomaclone(2D7-1F7)producing the anti-hTM4SF5EC2-CF peptide-specific monoclonal antibody was obtained with the standard hybridoma technique $(23,24)$. Five days after the final injection of a complex of hTM4SF5EC2-CF cyclic peptide and Lipoplex $(\mathrm{O})$, the mice $(\mathrm{n}=3)$ were anesthetized with isoflurane. Splenocytes producing the anti-hTM4SF5EC2-CF antibodies were extracted and hybridized with the SP2/0 mouse myeloma cell line (cat. no. CRL-1581; ATCC, Manassas, VA, USA). The 2D7-1F7 clone was selected by media containing hypoxanthine-aminopterin-thymidine (HAT) and hypoxanthine-thymidine $(\mathrm{HT})$. The remaining 5 of the $11 \mathrm{BALB} / \mathrm{c}\left(\mathrm{H}-2^{\mathrm{b}}\right)$ mice (weight, 20-22 g) were primed with pristine (Sigma-Aldrich, St. Louis, MO, USA; $0.5 \mathrm{ml} / \mathrm{mouse}$ ) and injected into the peritoneal cavity with the hybridoma cells (2D7-1H7 clone, $1 \times 10^{5}$ cells/mouse) for the production of ascitic fluid. Following the collection of ascitic fluid, the anti-hTM4SF5EC2-CF peptide-specific monoclonal antibody (mEC2-CF) was purified by Protein A column chromatography. The purified antibody was resolved by SDS-polyacrylamide gel electrophoresis (SDS-PAGE) using a $12.5 \%$ gel and confirmed by Coomassie Blue (cat. no. B0149; Sigma-Aldrich; 0.5 g/l) staining at room temperature for $1 \mathrm{~h}$.

Western blot analysis. To assess the ability of the monoclonal antibody to detect hTM4SF5, the 293F cells were transfected with hTM4SF5/pcDNA and 293F cells overexpressing
hTM4SF5 were selected (19). Cell lysates were prepared using a lysis buffer [10 mM HEPES (pH 8.4), $150 \mathrm{mM} \mathrm{NaCl}$, $5 \mathrm{mM}$ EDTA, $100 \mathrm{mM} \mathrm{NaF}, 2 \mathrm{mM} \mathrm{Na} \mathrm{VO}_{4}, 1 \%$ Nonidet P40 (NP-40) and protease inhibitor cocktail tablet (cat. no. 11697498001; Sigma-Aldrich)]. The protein concentration was measured using Copper(II) surface solution (cat. no. C2284) and bicinchoninic acid solution (cat. no. B9643) (both from Sigma-Aldrich). Equal amounts of proteins in the cell lysates were resolved by SDS-PAGE using $12.5 \%$ gel and electro-transferred onto nitro-cellulose membranes. The membranes were blocked with 5\% low-fat dry milk (Difco ${ }^{\mathrm{TM}}$ Skim Milk, cat. no. 232100; BD Biosciences, San Jose, CA, USA) in phosphate-buffered saline-Tween-20 (PBS-T; $140 \mathrm{mM} \mathrm{NaCl}, 2.7 \mathrm{mM} \mathrm{KCl}, 10 \mathrm{mM} \mathrm{Na} \mathrm{HPO}_{4}, 2 \mathrm{mM}$ $\mathrm{KH}_{2} \mathrm{PO}_{4}$ and $0.1 \%$ Tween-20) for $1 \mathrm{~h}$ at room temperature, and treated with mEC2-CF $(10 \mu \mathrm{g} / \mathrm{ml})$, a mouse anti-c-Myc tag antibody (1:1,000, cat. no. 2276; Cell Signaling Technology, Danvers, MA, USA), and monoclonal mouse anti- $\beta$-actin antibody (1:5,000, cat. no. A5316; Sigma-Aldrich) for $2 \mathrm{~h}$ at room temperature. Immunoreactive proteins were visualized by HRP-conjugated donkey anti-mouse $\operatorname{IgG}(1: 5,000$, cat. no. 715-035-150; Jackson-ImmunoResearch Laboratories) secondary antibody and an ECL solution (1:1 ratio, cat. nos. 1856135 and 1856136; Thermo Fisher Scientific) was performed for immunoprecipitation analyses.

Immunoprecipitation. Cell lysates were incubated with mEC2-CF $(10 \mu \mathrm{g} / \mathrm{ml})$ or mouse anti-c-Myc antibodies (1:500; Cell Signaling Technology) for $4 \mathrm{~h}$ at $4^{\circ} \mathrm{C}$ and then immunoprecipitated using Protein A beads. The samples were resolved using $12.5 \%$ SDS-PAGE and relevant proteins were detected using mEC2-CF and a mouse anti-Myc tag antibody $(1: 1,000$; Cell Signaling Technology). To assess whether mEC2-CF can detect the glycosylated form of hTM4SF5, lysates from hTM4SF5-overexpressing 293F cells were incubated with N-glycosidase F (PNGase F; Elpis-Biotech, Daejeon, Korea; cat. no. EBG-1005) in $1 \% \beta$-mercaptoethanol and $0.05 \%$ SDS for $1 \mathrm{~h}$ at $37^{\circ} \mathrm{C}$ and immunoprecipitated with mEC2-CF $(10 \mu \mathrm{g} / \mathrm{ml})$. The obtained immunocomplex was examined by $12.5 \%$ SDS-PAGE and western blot analysis.

Confocal images. To monitor antibody internalization, the HT-29, LoVo and HCT-116 cells were plated in 12-well plates and incubated with mouse normal $\mathrm{IgG}(1 \mu \mathrm{g} / \mathrm{ml}$, cat. no. 10400C; Thermo Fisher Scientific) and mEC2-CF $(1 \mu \mathrm{g} / \mathrm{ml})$ in RPMI-1640 medium containing 1\% FBS. After $3 \mathrm{~h}$, the cells were fixed with $4 \%$ formaldehyde for $10 \mathrm{~min}$, and blocked with PBS containing 3\% BSA and $0.1 \%$ Triton X-100 for $10 \mathrm{~min}$. The cells were then incubated with Alexa 488-conjugated goat anti-mouse IgG (ratio: 1:500, cat. no. A11001; Thermo Fisher Scientific) for $1 \mathrm{~h}$ at room temperature. To stain the nuclei, $10 \mu \mathrm{g} / \mathrm{ml}$ of Hoechst 33258 dye (Thermo Fisher Scientific) was added to the cells for $10 \mathrm{~min}$. After mounting with Fluoromount G (SouthernBiotech, Birmingham, AL, USA), the cells were scanned with a Carl Zeiss LSM 710 microscope (Carl Zeiss, Jena, Germany), in the Cooperative Center for Research Facilities, Hallym University (Chuncheon, Korea). To identify the co-localization of mEC2-CF and hTM4SF5, the HT-29 cells which express hTM4SF5 were incubated with Alexa 488-conjugated humanized anti-hTM4SF5 antibody 
(hEC2-C-2) recognizing different region of TM4SF5 (19) in RPMI-1640 medium containing 1\% FBS. After $3 \mathrm{~h}$, the cells were fixed with $4 \%$ formaldehyde for $10 \mathrm{~min}$, and blocked with PBS containing 3\% BSA and $0.1 \%$ Triton X-100 for $10 \mathrm{~min}$. The cells were incubated with mEC2-CF $(1 \mu \mathrm{g} / \mathrm{ml})$ and Alexa 594-conjugated goat anti-mouse IgG (1:500, cat. no. 53044; Thermo Fisher Scientific) for $1 \mathrm{~h}$ at room temperature.

MTT assay. The HT-29, LoVo and HCT-116 cells were plated in 24-well plates and the viability of the cells was examined with 3-(4,5-dimethylthiazol-2-yl)-2,5-diphenyltetrazolium bromide (MTT; Sigma-Aldrich). The cells were treated with mEC2-CF $(0,11,33$ and $100 \mathrm{nM})$ for 5 days. MTT solution was added to each well followed by incubation for $4 \mathrm{~h}$ at $37^{\circ} \mathrm{C}$, and the formazan crystals were dissolved in DMSO. The absorbance was read at $595 \mathrm{~nm}$ with a spectrophotometer (SpectraMAX 250; Molecular Devices, San Jose, CA, USA).

Patients and tissue samples. In this study, we included 204 patients with primary CRC who underwent radical surgical resection from November, 2006 to December, 2012 with no pre-operative chemotherapy or radiotherapy administered. All tissue samples were retrieved from the archive of the Department of Pathology, Hallym University Chuncheon Sacred Heart Hospital. Two independent pathologists (J.-Y.P. and K.C.C.) reviewed all the hematoxylin and eosin-stained tissue sample slides. Clinicopathological characteristics including tumor size, depth of invasion, histological grade, lymph node metastases, lymphatic invasion, vascular invasion and perineural invasion were assessed. Lymph node metastases were assessed in only 196 patients as the remaining 8 patients were diagnosed at the early stage of cancer and their lymph nodes were not dissected. All other clinicopathological characteristics were evaluated in the 204 patients with CRC. Patient age ranged from 19 to 87 years (mean and median, 67 years). During a mean follow-up period of 51 months (range, 2-113 months; median, 54 months), 15\% (27/186) of the patients with CRC succumbed to the disease. The survival of 18 patients was not evaluated as we could not contact the patients. Research protocols for the use of human tissues were approved by, and conducted in accordance with the policies of the Institutional Review Board at Hallym University Chuncheon Sacred Heart Hospital (Permit no. 2014-108). Informed consent was obtained from all study subjects.

Tissue microarray and immunohistochemistry. A tissue microarray was constructed with formalin-fixed, and paraffin-embedded tissue blocks from the 204 patients with CRC mentioned above. Representative sections from cases were selected through microscopic examination. Sample cores ( $2 \mathrm{~mm}$ in diameter) from each tissue block of CRC were inserted into recipient paraffin blocks in a grid pattern using a tissue arrayer (TMA-1; Unitma, Seoul, Korea). The slides were deparaffinized with xylene and rehydrated in ethanol, and endogenous peroxidase activity was blocked with $3 \%$ hydrogen peroxide for $15 \mathrm{~min}$. For antigen retrieval, all sections were boiled in a citrate buffer (pH 6.0; cat. no. CBB-500; ScyTek Laboratories, West Logan, UT, USA) for $15 \mathrm{~min}$. The slides were incubated overnight in PBST containing the mEC2-CF antibody $(10 \mu \mathrm{g} / \mathrm{ml})$ at $4^{\circ} \mathrm{C}$, followed by incubation with biotinylated anti-mouse IgG antibody (Histostain Plus kit; cat. no. 85-6643; Invitrogen, Carlsbad, CA, USA). They were sequentially treated with streptavidin-conjugated peroxidase, 3',3'-diaminobenzinidine $(0.5 \mathrm{mg} / \mathrm{ml})$, and $0.3 \%$ of hydrogen peroxide, and then counterstained with hematoxylin. After rinsing, the sections were mounted, dehydrated and covered with coverslips.

Evaluation of immunohistochemical staining. TM4SF5 expression was scored based on a semi-quantitative scoring method. Briefly, an intensity score was assigned as follows: 0 , negative staining; 1 , weak staining; 2 , moderate staining; or 3, strong staining; as was a proportion score as follows: 0 , none; $1,<25 \% ; 2,25-50 \% ; 3,50-75 \%$; and $4,>75 \%$. The products of the intensity and proportion scores were calculated to obtain the total score. We defined a high expression of TM4SF5 as a total score of $\geq 4$. All slides were examined and scored by two independent pathologists (J.-Y.P. and K.C.C.) who were blinded to the clinicopathological data and patient identity. Any discrepancy between assigned scores was resolved through re-examination by both pathologists to achieve a consensus.

Statistical analysis. The MTT assay results are shown as the means \pm standard deviation. Statistical significance of differences between 2 samples was evaluated using the Student's t-test; A value of $\mathrm{P}<0.05$ was considered to indicate a statistically significant difference. The association between TM4SF5 expression and clinicopathological characteristics was evaluated by Pearson's Chi-square test. The Kaplan-Meier test was used to analyze the survival rates and the significance of the difference in survival was determined using the log-rank test. Statistical significance was defined as a P-value $<0.05$. The SPSS statistical package version 15.0 (SPSS, Inc., Chicago, IL, USA) was used for statistical analysis.

\section{Results}

Selection of a candidate peptide and production of the mEC2-CF monoclonal antibody. Considering the structure of the hTM4SF5 EC2 domain and its functional implications (Fig. 1A), we designed a cyclic peptide, hTM4SF5EC2-CF (Fig. 1B). In particular, we designed the epitope focusing early part of the EC2 region by using several additionally extended amino acids in the $\mathrm{N}$-terminal to obtain a new type of antibody.

We immunized BALB/c mice with the hTM4SF5EC2-CF peptide and Lipoplex $(\mathrm{O})$ complex. When the sera were analyzed by ELISA, we found that the anti-hTM4SF5EC2-CF antibody had been successfully produced (Fig. 1C). Subsequently, 2D7-1F7 hybridoma clone cells producing the anti-hTM4SF5EC2-CF monoclonal antibody were selected and the production of the antibodies in the ascitic fluid of 5 mice was confirmed by ELISA (Fig. 2A). The antibody was separated and purified from the ascitic fluid by Protein A chromatography (Fig. 2B). ELISA identified the monoclonal antibody to be of the IgG2a isotype (Fig. 2C). To determine the specificity of the antibody, ELISA was performed with various TM4SF peptides (shown in Fig. 1B). The antibody exhibited strong binding to the hTM4SF5EC2-CF peptide, 

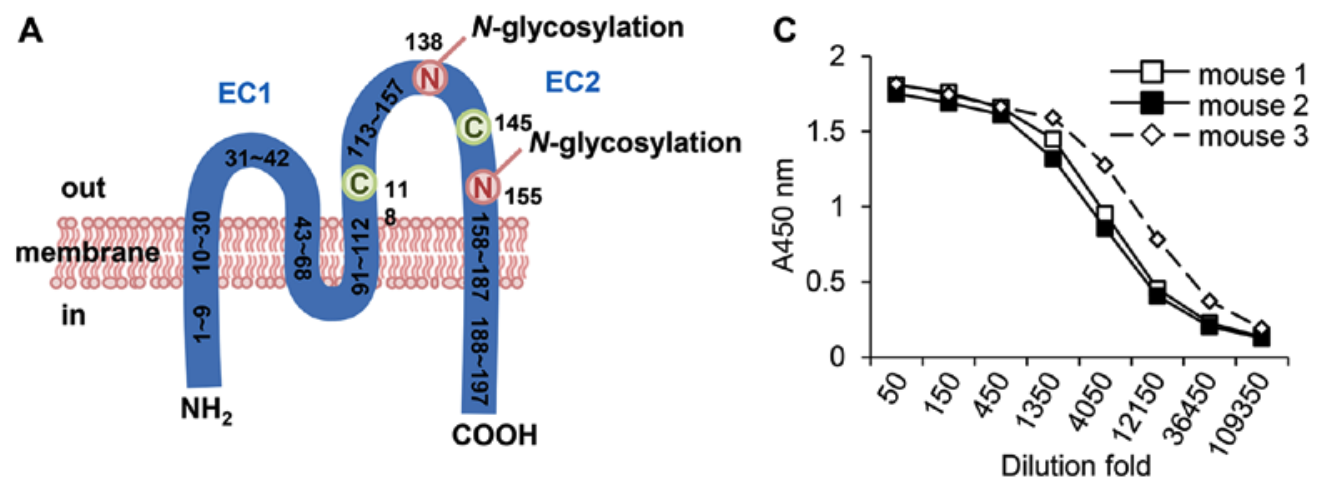

B

\begin{tabular}{cc}
\hline Abbreviations & Sequences \\
\hline mTM4SF5R2-3 & ${ }^{137}$ NDTLWNLCEAPPHV $^{150}$ \\
hTM4SF5R2-3 & ${ }^{138}$ NRTLWDRCEAPPRV $^{151}$ \\
hTM4SF5R2-N20T & ${ }^{138}$ NRTLWDRCEAPPRVVWNVT $^{157}$ \\
hTM4SF5R2-F25V & ${ }^{128}$ FEDTAGAYLLNRTLWDRCEAPPRVV $^{152}$ \\
hTM4SF5R2-G25T & ${ }^{133}$ GAYLLNRTLWDRCEAPPRVVWNVT $^{157}$ \\
hTM4SF5EC2-C & ${ }^{131}$ TACAYLLNRTLWDRSEAPPRVVPWNCT $^{157}$ \\
hTM4SF5EC2-CF & ${ }^{116}$ PRCLMNGEWGYHFEDTAGAYLLNRTLWDRCEAPPRVVP ${ }^{153}$ \\
\hline
\end{tabular}

Figure 1. Production of the hTM4SF5EC2-CF peptide-specific antibody. (A) Structure of hTM4SF5 in the membrane. (B) Location of hTM4SF5 peptide epitopes. (C) The B-cell peptide epitope of TM4SF5 (hTM4SF5EC2-CF) and Lipoplex(O) complex was used to immunize BALB/c mice and sera were analyzed by ELISA using hTM4SF5EC2-CF peptide as an antigen.
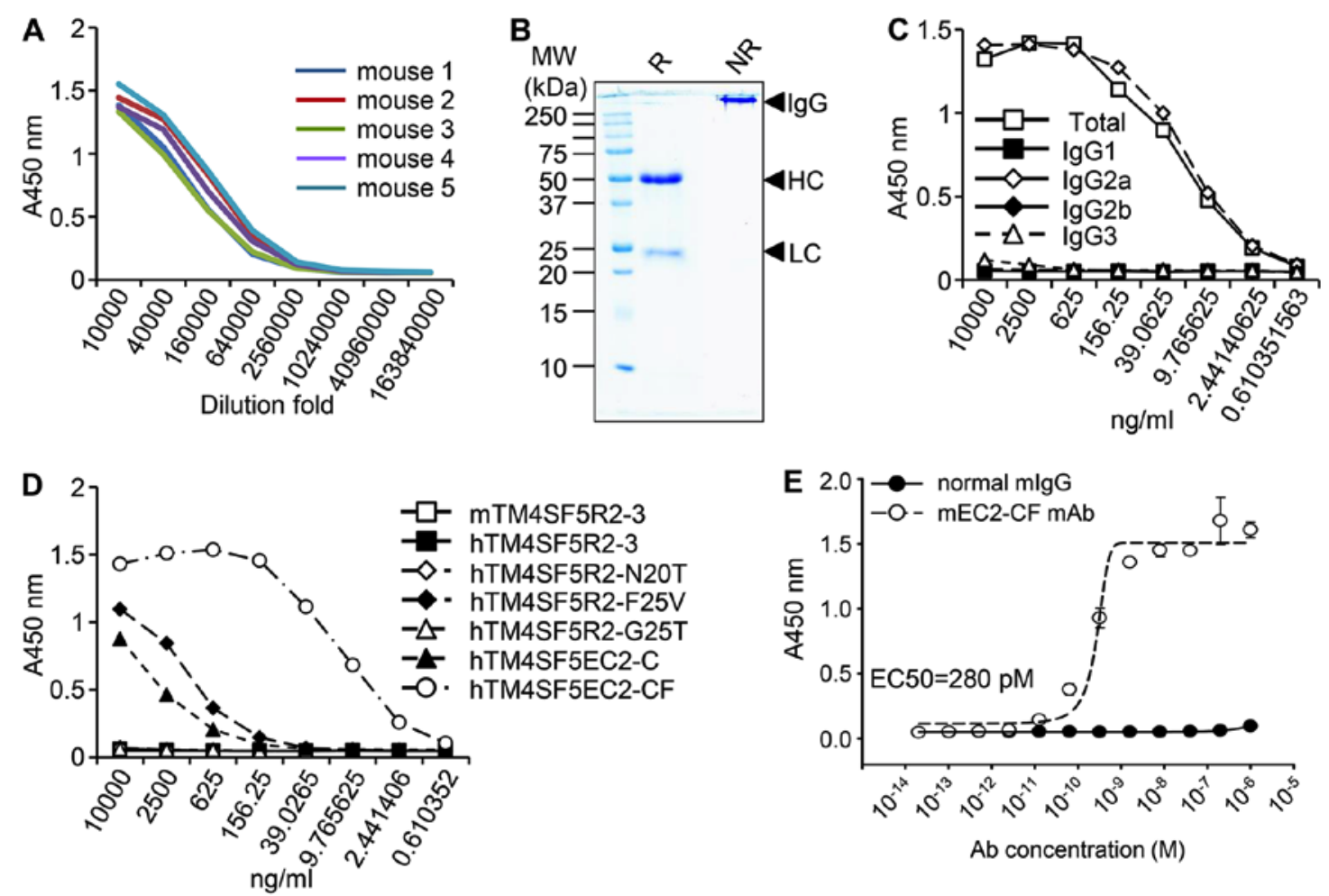

Figure 2. Purification and characterization of the anti-hTM4SF5EC2-CF peptide-specific monoclonal antibody. (A) Ascitic fluid was obtained from 2D7-1F7 clone-injected mice and production of the hTM4SF5EC2-CF peptide-specific monoclonal antibody was analyzed by ELISA. (B) The anti-hTM4SF5EC2-CF peptide-specific monoclonal antibody (mEC2-CF) was purified by Protein A column chromatography and detected using SDS-PAGE and Coomassie Blue staining. R, reducing condition; NR, Non-reducing condition; HC, heavy chain; LC, light chain. (C) To determine the isotype of the antibody, isotype-specific ELISA was performed. (D) Determination of the binding specificity of the antibody to hTM4SF5EC2-CF peptide. (E) Measurement of binding affinity of the antibody with hTM4SF5EC2-CF peptide using ELISA.

very weak binding to two other peptides (hTM4SF5R2-F25V and hTM4SF5EC2-C) at high concentrations (Fig. 2D), and no binding to the other peptides tested. These results suggest that the monoclonal antibody is highly specific to 

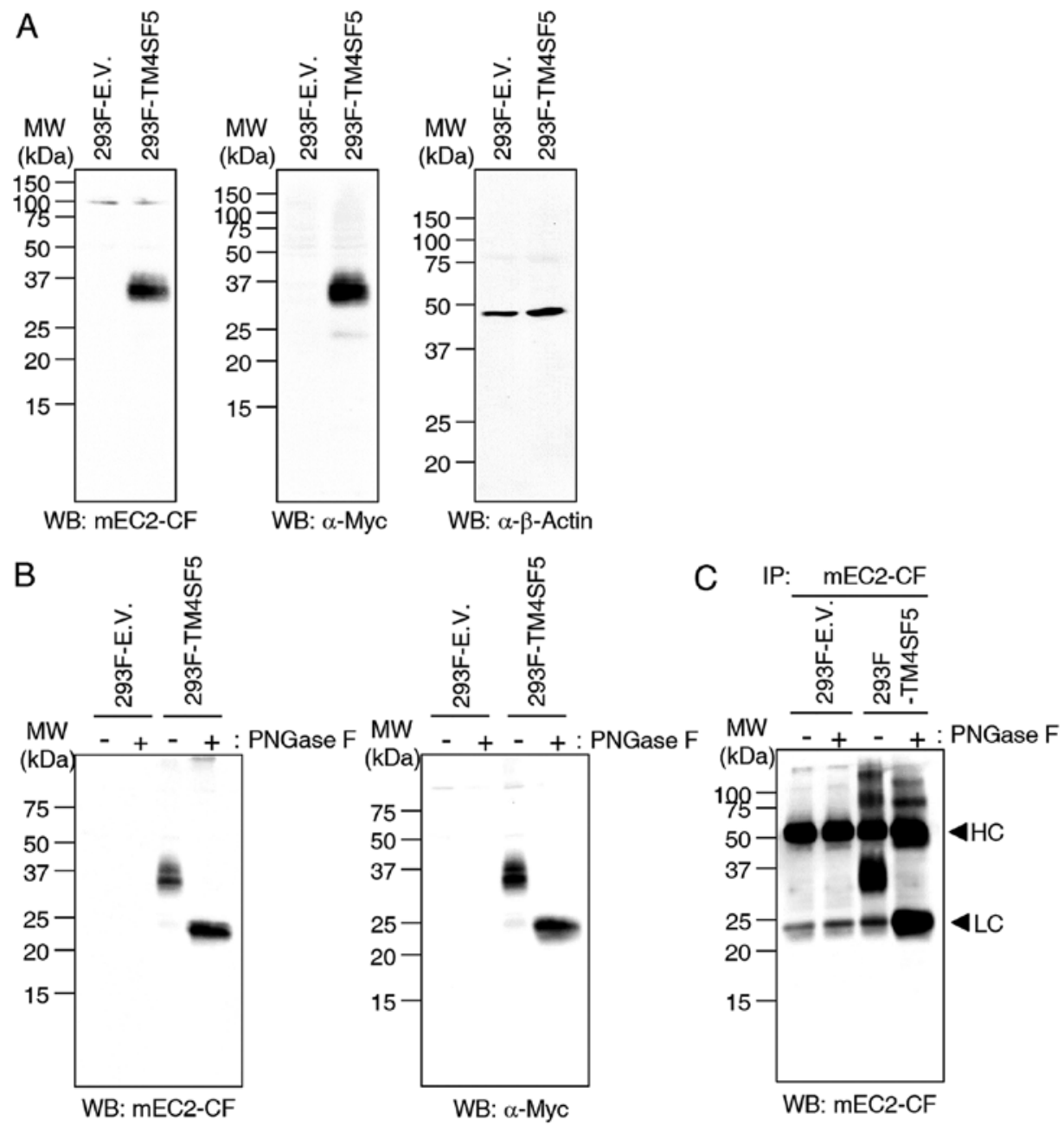

Figure 3. Reactivity of the anti-hTM4SF5EC2-CF peptide-specific monoclonal antibody. The empty vector or a TM4SF5 expression vector was transfected into $293 \mathrm{~F}$ cells. (A) Cell lysates were examined by western blot analysis with mEC2-CF, rabbit anti-Myc antibody, or anti- $\beta$-actin antibody. (B) Cell lysates were treated with N-glycosidase F (PNGase F) and examined by western blot analysis using mEC2-CF or rabbit anti-Myc antibody. (C) Cell lysates were treated with $\mathrm{N}$-glycosidase $\mathrm{F}$ (PNGase F) and immunoprecipitated with mEC2-CF and examined by western blot analysis using mEC2-CF. HC, heavy chain; LC, light chain.

hTM4SF5EC2-CF, which possibly mimics the structure of the natural EC2 region of TM4SF5. The antibody binding affinity was evaluated and the EC50 was 280 pM (Fig. 2E). These results demonstrated that the hTM4SF5EC2-CF peptide epitope induced production of an IgG2a-type antibody with strong and specific affinity towards the hTM4SF5EC2-CF peptide. The anti-hTM4SF5EC2-CF monoclonal antibody was named mEC2-CF.

Characterization of $m E C 2-C F$. To investigate the function of mEC2-CF, 293F cells were transfected with empty vector or Myc-tagged hTM4SF5 (hTM4SF5-myc) expression vector and the cell lysates were examined by western blot analysis and immunoprecipitation using the mEC2-CF antibody. The results of western blot analysis demonstrated that mEC2-CF effectively recognizes hTM4SF5-myc protein in cell lysates and the specificity was confirmed using an anti-Myc antibody (Fig. 3A). We then de-glycosylated hTM4SF5-myc protein by treating the cell lysates with N-glycosidase F (PNGase F) and examined the reactivity of $\mathrm{mEC} 2-\mathrm{CF}$ by western blot analysis and immunoprecipitation. When the cell lysates were treated with PNGase $\mathrm{F}$, the mobility of the detected protein band was enhanced compared to the untreated control. Considering that TM4SF5 has two $\mathrm{N}$-glycosylation sites, the more rapidly moving protein band seemed to be unglycosylated hTM4SF5-myc. It was also confirmed by western blot analysis with an anti-Myc antibody (Fig. 3B). Therefore, these results suggested that the mEC2-CF antibody could detect both glycosylated and unglycosylated hTM4SF5-myc protein. mEC2-CF was also able to immunoprecipitate both forms of hTM4SF5-myc protein, which was confirmed by western blot analysis with mEC2-CF (Fig. 3C). When the cell lysates were treated with PNGase F, the protein band of unglycosylated hTM4SF5-myc protein was detected to be overlapped with the light chain of antibody due to their similar mobility. Therefore, we concluded that $\mathrm{mEC} 2-\mathrm{CF}$ is reactive to the hTM4SF5 protein and that the glycosylation of Asn at 138 of hTM4SF5 is likely not required for the recognition of hTM4SF5 by the mEC2-CF antibody.

Internalization of TM4SF5 bound by mEC2-CF. Considering the antibody-drug conjugate strategy, the ability of an antibody to internalize into cells is an important parameter for the therapeutic efficacy. In this study, to determine whether mEC2-CF could be internalized into cells, HT-29, HCT-116 


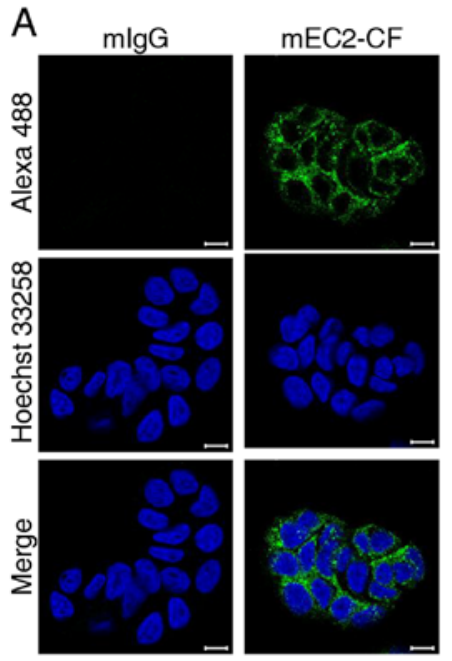

HT-29
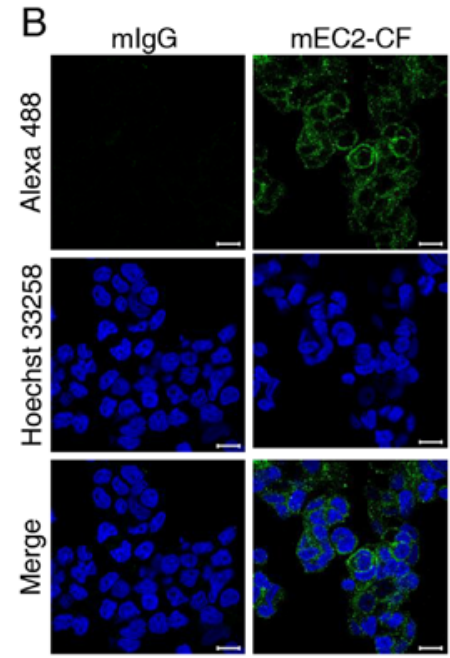

LoVo

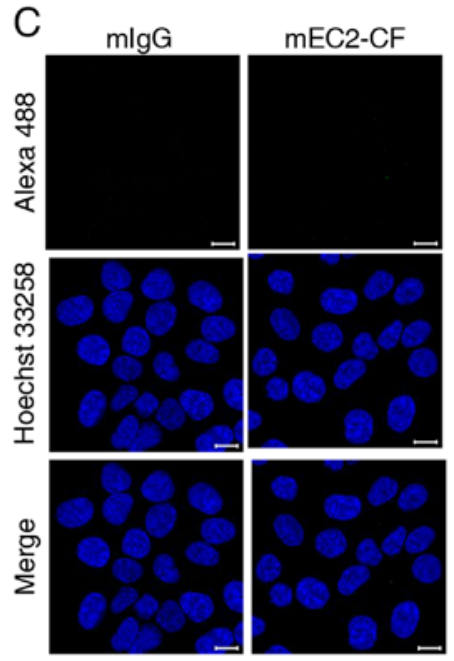

HCT-116

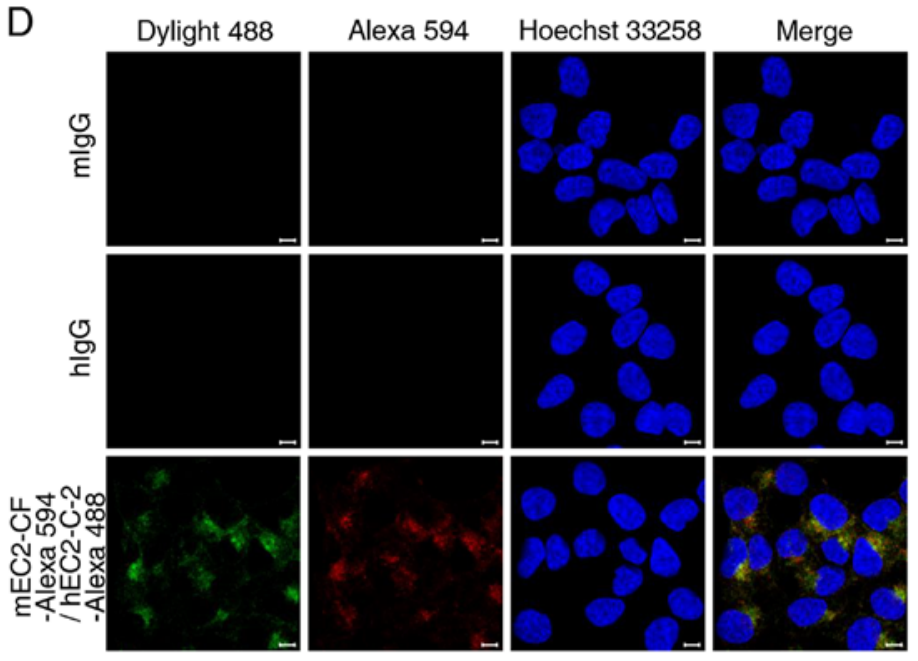

E HT-29

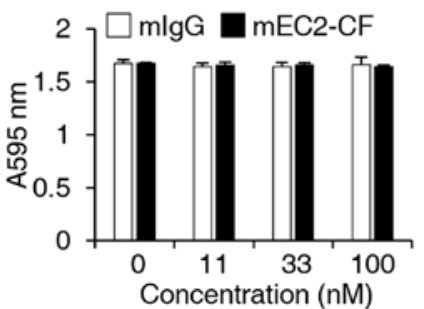

LoVo

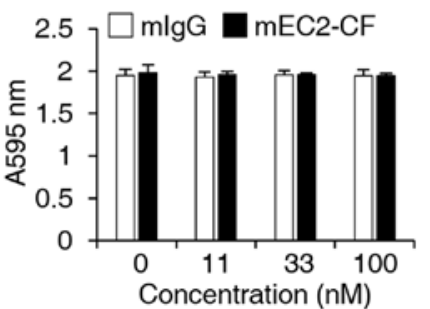

HCT-116

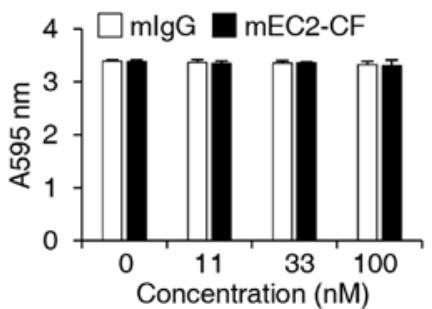

Figure 4. Internalization of TM4SF5 in colorectal cancer (CRC) cells. The CRC cells, (A) HT-29, (B) LoVo and (C) HCT-116 were incubated with mEC2-CF or mouse normal $\mathrm{IgG}(\mathrm{mIgG})$ for $3 \mathrm{~h}$ followed by incubation with an Alexa 488-conjugated secondary antibody. (D) The HT-29 cells were incubated with Alexa 488-conjugated hEC2-C-2, mouse normal IgG (mIgG) or human normal $\operatorname{IgG}(\mathrm{hIgG})$ for $3 \mathrm{~h}$ and then incubated with $\mathrm{mEC} 2-\mathrm{CF}$ followed by incubation with an Alexa 594-conjugated anti-mouse secondary antibody. For nuclear staining, the cells were incubated with Hoechst 33258. Images were captured using a confocal microscope. Scale bar, $20 \mu \mathrm{m}$. (E) No effect of mEC2-CF was observed on cell viability. The HT-29, LoVo and HCT-116 cells were untreated (0), or treated with 11, 33 or $100 \mathrm{nM}$ of mEC2-CF for 5 days followed by MTT assay. The data are presented as the means \pm standard deviation. The differences between two samples were analyzed using the Student's t-test.

and LoVo cells were treated with mEC2-CF and then incubated with Alexa 488-conjugated goat anti-mouse $\mathrm{IgG}$. Immunofluorescence analysis using confocal microscopy revealed that mEC2-CF was successfully internalized into the HT-29 and LoVo cells, which express hTM4SF5 $(18,19)$ (Fig. 4A and B). No such internalization was observed in the HCT-116 cells, which do not express hTM4SF5 (19), suggesting that the internalization of mEC2-CF is mediated by its binding to hTM4SF5 (Fig. 4C). To further confirm that the internalization is mediated by targeting hTM4SF5, the HT-29 cells were incubated with Alexa 488-conjugated humanized anti-TM4SF5 antibody (hEC2-C-2) recognizing another epitope of EC2 (19). After $3 \mathrm{~h}$, the cells were fixed, permeabilized and then stained with mEC2-CF and Alexa 594-conjugated goat anti-mouse IgG. As shown in Fig. 4D, both the antibodies were co-localized in the confocal images. Based on these observations, we concluded that mEC2-CF effectively recognizes hTM4SF5 and then internalizes into hTM4SF5 protein-expressing target cells. To further identify the biological effect of mEC2-CF on cell growth, the viability of the CRC cells in the presence 


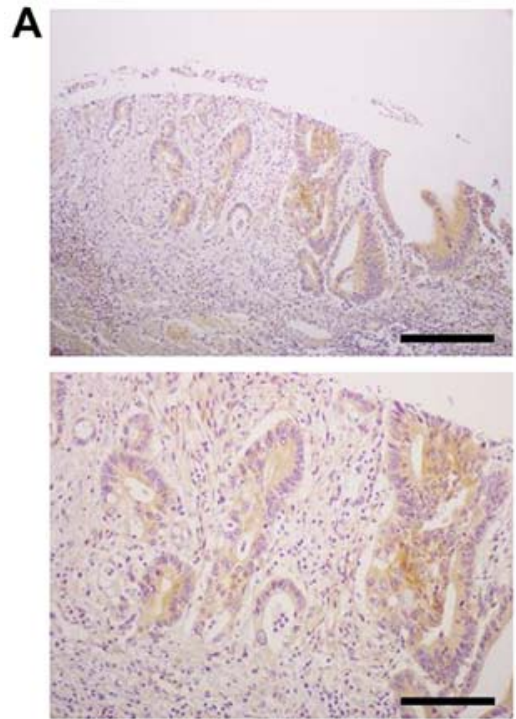

Low TM4SF5 expression
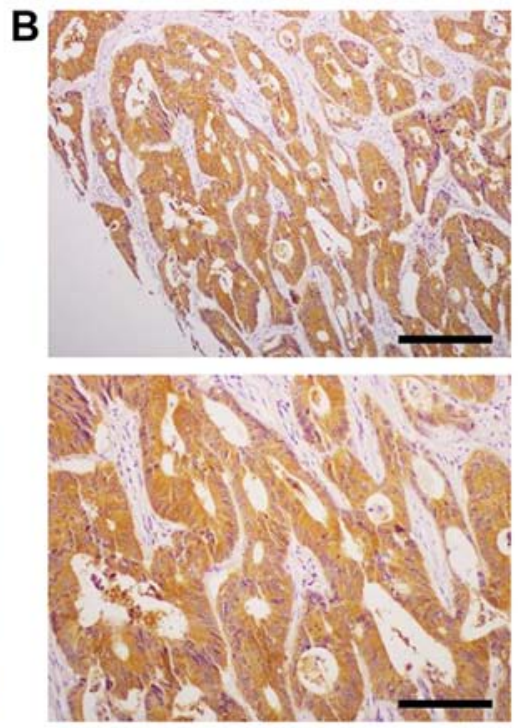

High TM4SF5 expression

Figure 5. Immunohistochemistry of the tissue samples from patients with colorectal cancer (CRC). The tissue samples were examined by immunohistochemical staining with mEC2-CF. Based on the staining intensity and percentage of stained cells, the samples were classified into two groups of low and high TM4SF5 expression. Representative samples with (A) low and (B) high TM4SF5 expression are shown. Images were captured under a microscope. Upper panel scale bar, $100 \mu \mathrm{m}$; lower panel scale bar, $25 \mu \mathrm{m}$.
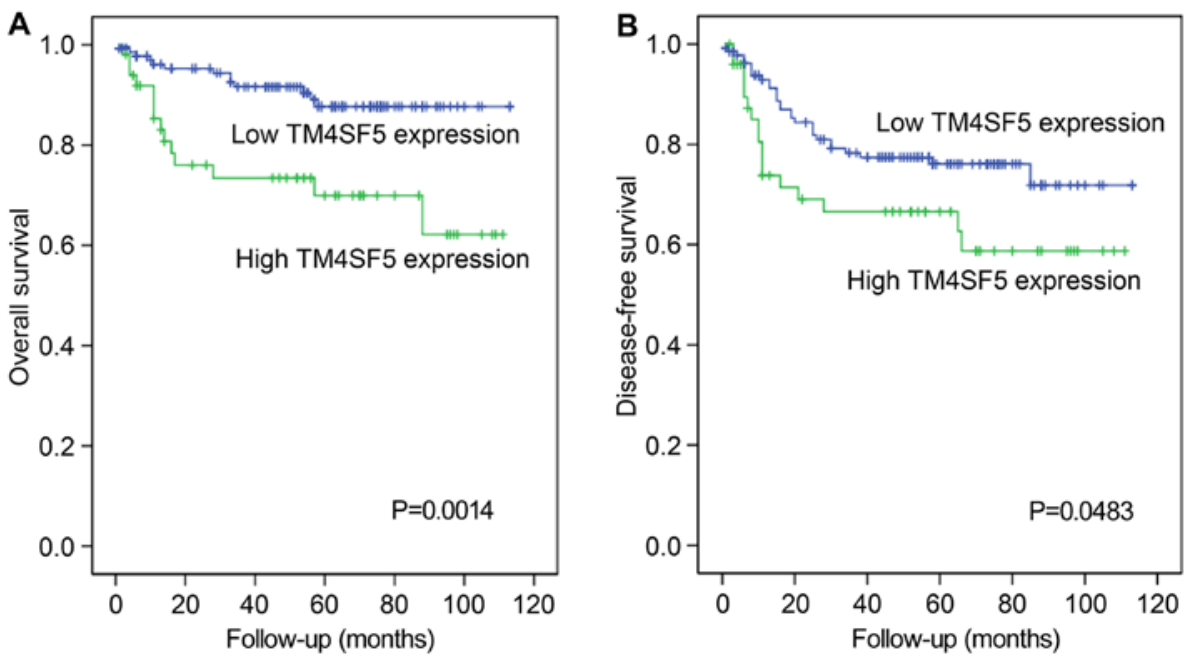

Figure 6. Reciprocal association of TM4SF5 expression with overall and disease-free survival rates of patients with colorectal cancer. Overall survival and disease-free survival for 10 years of the 2 groups with a low or high expression of TM4SF5 were plotted and analyzed using the Kaplan-Meier method.

of mEC2-CF was examined but there was no significant effect (Fig. 4E).

Detection of TM4SF5 protein expression in CRC tissues with $m E C 2-C F$. To determine the effect of hTM4SF5 expression on the clinicopathological characteristics of patients with CRC, we examined the protein expression of hTM4SF5 in CRC tissues using immunohistochemistry with mEC2-CF (Fig. 5). A high expression of hTM4SF5 was detected in 55 of the 204 CRC cases $(27 \%)$. We then examined whether TM4SF5 expression is associated with tumor size, depth of invasion, histological grade, lymph node metastases, lymphatic invasion, vascular invasion and perineural invasion. The patients with a high TM4SF5 expression exhibited significantly increased tumor invasion compared with those with a low TM4SF5 expression
$(\mathrm{P}=0.027)$. Other clinicopathological characteristics evaluated were not associated with TM4SF5 expression (Table I).

Survival analysis. To investigate the possible involvement of hTM4SF5 expression in the prognosis of patients with CRC, Kaplan-Meier tests for overall survival and disease-free survival were performed. As shown in Fig. 6, a high expression of hTM4SF5 was significantly associated with a worse overall survival $(\mathrm{P}=0.0014)$ and disease-free survival $(\mathrm{P}=0.0483)$ of patients with CRC.

In addition, our analysis revealed that the decreased patient survival was significantly associated with the depth of invasion $(\mathrm{P}=0.0227)$, lymphatic invasion $(\mathrm{P}=0.0047)$, lymph node metastasis $(\mathrm{P}=0.002)$ and perineural invasion $(\mathrm{P}=0.0005)$. Worse disease-free survival was also significantly associated 
Table I. The association between TM4SF5 and the clinicopathological characteristics of patients with colorectal cancer.

\begin{tabular}{|c|c|c|c|}
\hline \multirow[b]{2}{*}{$\begin{array}{l}\text { Clinicopathological } \\
\text { characteristics }\end{array}$} & \multicolumn{2}{|c|}{ TM4SF5 expression } & \multirow[b]{2}{*}{ P-value } \\
\hline & $\begin{array}{c}\text { Low } \\
(\mathrm{n}=149)\end{array}$ & $\begin{array}{l}\text { High } \\
(n=55)\end{array}$ & \\
\hline \multicolumn{4}{|l|}{ Tumor size } \\
\hline$\leq 5 \mathrm{~cm}$ & $86(74.1 \%)$ & $30(25.9 \%)$ & 0.685 \\
\hline$>5 \mathrm{~cm}$ & $63(71.6 \%)$ & $25(28.4 \%)$ & \\
\hline \multicolumn{4}{|l|}{ Depth of invasion } \\
\hline $\mathrm{T} 1$ and $\mathrm{T} 2$ & $41(85.4 \%)$ & $7(14.6 \%)$ & $0.027^{\mathrm{a}}$ \\
\hline $\mathrm{T} 3$ and $\mathrm{T} 4$ & $108(69.2 \%)$ & $48(30.8 \%)$ & \\
\hline \multicolumn{4}{|l|}{ Histological grade } \\
\hline WD & $30(78.9 \%)$ & $8(21.1 \%)$ & 0.343 \\
\hline MD and PD & $119(71.7 \%)$ & $47(28.3 \%)$ & \\
\hline \multicolumn{4}{|l|}{ Lymphatic invasion } \\
\hline Present & $58(72.5 \%)$ & $22(27.5 \%)$ & 0.889 \\
\hline Absent & $91(73.4 \%)$ & $33(26.6 \%)$ & \\
\hline \multicolumn{4}{|l|}{ Vascular invasion } \\
\hline Present & $141(73.8 \%)$ & $50(26.2 \%)$ & 0.343 \\
\hline Absent & $8(61.5 \%)$ & $5(38.5 \%)$ & \\
\hline \multicolumn{4}{|l|}{ Perineural invasion } \\
\hline Present & $106(71.1 \%)$ & $43(28.9 \%)$ & 0.315 \\
\hline Absent & $43(78.2 \%)$ & $12(21.8 \%)$ & \\
\hline \multicolumn{4}{|c|}{ Lymph node metastasis ${ }^{\mathrm{b}}$} \\
\hline Present & $87(72.5 \%)$ & $33(27.5 \%)$ & 0.984 \\
\hline Absent & $55(72.4 \%)$ & $21(27.6 \%)$ & \\
\hline
\end{tabular}

Data were analyzed using Pearson's Chi-square test. ${ }^{\mathrm{a}} \mathrm{P}<0.05$, indicating statistical significance; ${ }^{\text {it }}$ should be noted that lymph node metastases were assessed in only 196 patients. WD, well differentiated; MD, moderately differentiated; PD, poorly differentiated.

with the depth of invasion $(\mathrm{P}=0.0221)$, lymphatic invasion $(\mathrm{P}=0.0081)$, vascular invasion $(\mathrm{P}<0.0001)$, lymph node metastasis $(\mathrm{P}<0.0001)$ and perineural invasion $(\mathrm{P}=0.0009)$ (Table II). These data are in accordance with current knowledge regarding the prognosis of patients with CRC.

\section{Discussion}

Previously, we screened an efficacious B-cell peptide epitope (hTM4SF5R2-3) by immunizing BALB/c mice with various TM4SF5 peptides and characterized the prophylactic and the therapeutic effects of the peptide vaccine on hepatocellular carcinoma and colon cancer cell lines and tumor mouse models $(8,9,25)$. We also produced TM4SF5-specific monoclonal antibodies and evaluated their therapeutic effects $(18,19,26)$. Based on these data, we hypothesized that the hTM4SF5 protein is a reasonable target for anticancer therapy and that anti-hTM4SF5 antibodies warrant further development as therapeutic agents for the inhibition of hTM4SF5 function in cancer. To isolate more effective monoclonal antibodies targeting hTM4SF5, we recently isolated an anti-hTM4SF5 monoclonal
Table II. Univariate analyses for the overall survival and disease-free survival of patients with colorectal cancer.

\begin{tabular}{lcc}
\hline $\begin{array}{l}\text { Clinicopathological } \\
\text { characteristics }\end{array}$ & $\begin{array}{c}\text { Overall survival } \\
\text { P-value }\end{array}$ & $\begin{array}{c}\text { Disease-free } \\
\text { survival } \\
\text { P-value }\end{array}$ \\
\hline Size & 0.4708 & 0.4817 \\
Depth of invasion & $0.0227^{\mathrm{a}}$ & $0.0021^{\mathrm{a}}$ \\
Lymphatic invasion & $0.0047^{\mathrm{a}}$ & $0.0081^{\mathrm{a}}$ \\
Vascular invasion & 0.1555 & $<0.0001^{\mathrm{a}}$ \\
Perineural invasion & $0.0005^{\mathrm{a}}$ & $0.0009^{\mathrm{a}}$ \\
Histological grade & 0.0769 & 0.0716 \\
Lymph node metastasis & $0.0002^{\mathrm{a}}$ & $<0.0001^{\mathrm{a}}$ \\
\hline
\end{tabular}

${ }^{\mathrm{a}} \mathrm{P}<0.05$, indicating statistical significance.

antibody using a cyclic peptide that mimics the native EC2 region (hTM4SF5EC2-C) and characterized the anti-metastatic effects of its humanized antibody in a colon cancer model (19). In this study, we produced and verified a monoclonal antibody (mEC2-CF) against another cyclic peptide epitope targeting the EC2 domain (hTM4SF5EC2-CF) of hTM4SF5.

The monoclonal antibody, mEC2-CF, was highly specific to the cyclic peptide hTM4SF5EC2-CF, with low cross-reactivity to two other TM4SF5 peptides, possibly due to the partial overlap of the epitopes. It is hard to determine the exact epitope of the antibody due to the complexity involving structure and sequence. Considering that two glycosylation sites (N138 and N155) are known to be essential for the function of TM4SF5 (16) and that mEC2-CF cannot inhibit the growth of TM4SF5-expressing cells, it is likely that the antibody recognizes the early part of EC2 region rather than the late part of EC2, including the glycosylation sites as we originally planned.

The antibody recognized recombinant hTM4SF5 protein produced by ectopic over-expression in $293 \mathrm{~F}$ cells, as well as endogenous hTM4SF5 protein overexpressed in colon cancer cell lines. Therefore, we postulated that this antibody may be useful for detection of hTM4SF5 in clinical samples of patients with CRC. The results of immunohistochemistry revealed that mEC2-CF successfully recognized hTM4SF5 in the colon tissues from patients with CRC. When we performed immunohistochemistry on the clinical samples with the previously developed anti-hTM4SF5 antibody targeting hTM4SF5EC2-C, we did not obtain the expected results. One important thing to be considered is that the isotype of the antibody is IgG3 and IgG3 antibodies are generally unstable. In the case of its humanized antibody, it is not suitable for the staining of human tissues. Accounting that the isotype of mEC2-CF is IgG2a and the target epitope seems different from the original one, we tried to use this antibody in immunohistochemistry to analyze TM4SF5 expression in clinical samples and found that the new antibody has a unique advantage for the purposes of immunohistochemistry.

Our analysis also revealed that $\mathrm{mEC} 2-\mathrm{CF}$ can be internalized after binding to hTM4SF5, further supporting the utility of this antibody in development of cancer therapy. However, in contrast to previously-isolated antibodies, mEC2-CF did not reduce the growth of hTM4SF5-expressing cells, suggesting 
that the antibody binds to hTM4SF5, but may not inhibit the function of hTM4SF5 in cells. Therefore, this antibody may have limited utility when applied as a single agent in the treatment of cancer. However, given the specificity of its targeting and its internalization into cells, the development of antibody-drug conjugates of mEC2-CF may be an alternative strategy for its application in anticancer therapeutics.

Previously, we demonstrated a higher expression of hTM4SF5 in colon cancer tissues compared to normal tissues by analyzing commercially-available tissue microarrays using immunohistochemistry (18). The vendor provided information regarding sex, age, tumor grade, along with the tumor, node and metastasis (TNM) stage of the tissues included in the microarray. However, there was no information regarding the prognosis of the patients. In this study, we analyzed the association between hTM4SF5 expression and the prognosis of patients with CRC after surgery using the newly-isolated mEC2-CF antibody and tumor tissues obtained from CRC patients. The results clearly revealed that a high expression of hTM4SF5 in the cancer tissues was associated with a poor prognosis, represented by the low overall and disease-free survival rates. Therefore, we suggest that hTM4SF5 expression is suitable as a marker for a poor prognosis in CRC.

To date, we have isolated several monoclonal antibodies against hTM4SF5 and produced one humanized antibody. In this study, we isolated and characterized another candidate antibody and verified its utility as a tool to investigate hTM4SF5 expression. Different antibodies have different characteristics as we expected. For example, the anti-hTM4SF5 antibody targeting hTM4SF5EC2-C was effective in the inhibition of tumor growth in vitro and in vivo (19). By contrast, mEC2-CF did not inhibit the growth of cancer cells in vitro, although it proved to be useful in detection using immunohistochemistry. Therefore, we consider that obtaining more diverse candidate antibodies may provide us with an array of choices when considering antibodies for diagnostic, prognostic and therapeutic applications in cancer treatment. For future application, the in vivo stability and efficacy of the candidate antibodies will have to be determined in detail and strategies to strengthen the efficacy of antibodies in the inhibition of tumor growth will have to be evaluated and verified.

\section{Acknowledgements}

Not applicable.

\section{Funding}

This study was supported by grants from the National Research Foundation (2013M3A9A9050126, 2015R1A2A2A01007209, 2016M3A9B6916708 and 2009-0093812) funded by the Ministry of Science and ICT in the Republic of Korea. BKP was supported by the Hallym University Postdoctoral Fellowship Program of 2017 (HLM-PF-2017-0001).

\section{Availability of data and materials}

All data generated or analyzed during this study are included in this published article.

\section{Authors' contributions}

YL, HJK and KCC conceived the study and its design and wrote the manuscript. BKP, JYP, THK, DK, GW, AG, SM and SIL performed the experiments. JYP and KCC performed the clinicopathological analysis. BKP, THK, DK, GW and SIL were involved in the animal experiments. BKP, JYP, YL, HJK and $\mathrm{KCC}$ performed the revision of the manuscript and edited the manuscript. All authors have read and approved the final manuscript.

\section{Ethics approval and consent to participate}

Research protocols for the use of human tissues were approved by, and conducted in accordance with the policies of the Institutional Review Board at Hallym University Chuncheon Sacred Heart Hospital (Permit no. 2014-108). Informed consent was obtained from all study subjects. the animal experiments were approved by the Institutional Animal Care and Use Committee of Hallym University (Permit no. Hallym 2015-55, Hallym 2015-56).

\section{Consent for publication}

Not applicable.

\section{Competing interests}

The authors declare that they have no competing interests.

\section{References}

1. World Health Organization: 'GLOBOCAN 2012: Estimated Cancer Incidence, Mortality and Prevalence Worldwide in 2012'. http://globocan.iarc.fr/Pages/fact_sheets_cancer. aspx. Retrieved April, 2015.

2. Wilkinson $\mathrm{N}$ and Scott-Conner CE: Surgical therapy for colorectal adenocarcinoma. Gastroenterol Clin North Am 37: 253-267, 2008.

3. Goodwin RA and Asmis TR: Overview of systemic therapy for colorectal cancer. Clin Colon Rectal Surg 22: 251-256, 2009.

4. Müller-Pillasch F, Wallrapp C, Lacher U, Friess H, Büchler M, Adler G and Gress TM: Identification of a new tumour-associated antigen TM4SF5 and its expression in human cancer. Gene 208: 25-30, 1998.

5. Lee SA, Lee SY, Cho IH, Oh MA, Kang ES, Kim YB, Seo WD, Choi S, Nam JO, Tamamori-Adachi M, et al: Tetraspanin TM4SF5 mediates loss of contact inhibition through epithelial-mesenchymal transition in human hepatocarcinoma. J Clin Invest 118: 1354-1366, 2008.

6. Lee SA, Ryu HW, Kim YM, Choi S, Lee MJ, Kwak TK, Kim HJ, Cho M, Park KH and Lee JW: Blockade of four-transmembrane L6 family member 5 (TM4SF5)-mediated tumorigenicity in hepatocytes by a synthetic chalcone derivative. Hepatology 49: 1316-1325, 2009.

7. Kwon S, Kim D, Park BK, Cho S, Kim KD, Kim YE, Park CS, Ahn HJ, Seo JN, Choi KC, et al: Prevention and therapy of hepatocellular carcinoma by vaccination with TM4SF5 epitopeCpG-DNA-liposome complex without carriers. PLoS One 7: e33121, 2012.

8. Kwon S, Kim D, Park BK, Wu G, Park MC, Ha YW, Kwon HJ and Lee Y: Induction of immunological memory response by vaccination with TM4SF5 epitope-CpG-DNA-liposome complex in a mouse hepatocellular carcinoma model. Oncol Rep 29: 735-740, 2013.

9. Kwon S, Kim YE, Kim D, Park BK, Wu G, Kim TH, Choi SH, Kim DS, Kwon HJ and Lee Y: Prophylactic effect of a peptide vaccine targeting TM4SF5 against colon cancer in a mouse model. Biochem Biophys Res Commun 435: 134-139, 2013. 
10. Wu YB, Huang YS, Xu YP, Sun YF, Yu DL, Zhang XQ, Long X, Zhu SQ, Zhou JL and Xu JJ: A high level of TM4SF5 is associated with human esophageal cancer progression and poor patient survival. Dig Dis Sci 58: 2623-2633, 2013.

11. Wright MD, Ni J and Rudy GB: The L6 membrane proteins - a new four-transmembrane superfamily. Protein Sci 9: 1594-1600, 2000.

12. Detchokul S, Williams ED, Parker MW and Frauman AG: Tetraspanins as regulators of the tumour microenvironment: Implications for metastasis and therapeutic strategies. Br J Pharmacol 171: 5462-5490, 2014.

13. Yáñez-Mó M, Barreiro O, Gordon-Alonso M, Sala-Valdés M and Sánchez-Madrid F: Tetraspanin-enriched microdomains: A functional unit in cell plasma membranes. Trends Cell Biol 19: 434-446, 2009.

14. Jung O, Choi S, Jang SB, Lee SA, Lim ST, Choi YJ, Kim HJ, Kim DH, Kwak TK, Kim H, et al: Tetraspan TM4SF5-dependent direct activation of FAK and metastatic potential of hepatocarcinoma cells. J Cell Sci 125: 5960-5973, 2012.

15. Kim HJ, Kwon S, Nam SH, Jung JW, Kang M, Ryu J, Kim JE, Cheong JG, Cho CY, Kim S, et al: Dynamic and coordinated single-molecular interactions at TM4SF5-enriched microdomains guide invasive behaviors in 2- and 3-dimensional environments. FASEB J 31: 1461-1481, 2017.

16. Lee SA, Kim YM, Kwak TK, Kim HJ, Kim S, Ko W, Kim SH, Park KH, Kim HJ, Cho M, et al: The extracellular loop 2 of TM4SF5 inhibits integrin alpha2 on hepatocytes under collagen type I environment. Carcinogenesis 30: 1872-1879, 2009.

17. Kwon S, Choi KC, Kim YE, Ha YW, Kim D, Park BK, Wu G Kim DS, Lee Y and Kwon HJ: Monoclonal antibody targeting of the cell surface molecule TM4SF5 inhibits the growth of hepatocellular carcinoma. Cancer Res 74: 3844-3856, 2014.
18. Kim YE, Kwon S, Wu G, Kim D, Park BK, Park JA, Choi KC, Kim DS, Kwon HJ and Lee Y: Therapeutic effect of a TM4SF5specific monoclonal antibody against colon cancer in a mouse model. Oncotarget 5: 8402-8415, 2014.

19. Wu G, Kim D, Park BK, Park S, Ha JH, Kim TH, Gautam A, Kim JN, Lee SI, Park HB, et al: Anti-metastatic effect of the TM4SF5-specific peptide vaccine and humanized monoclonal antibody on colon cancer in a mouse lung metastasis model. Oncotarget 7: 79170-79186, 2016.

20. Kim D, Kwon S, Ahn CS, Lee Y, Choi SY, Park J, Kwon HY and Kwon HJ: Adjuvant effect of liposome-encapsulated natural phosphodiester CpG-DNA. BMB Rep 44: 758-763, 2011.

21. Park BK, Choi SH, Kim YE, Park S, Lee Y, Lee KW and Kwon HJ: Monoclonal antibodies against the human respiratory syncytial virus obtained by immunization with epitope peptides and CpG-DNA-liposome complex. Monoclon Antib Immunodiagn Immunother 34: 101-109, 2015.

22. Park BK, Lee SI, Lee Y and Kwon HJ: Effect of a monoclonal antibody against human relaxin-2 on cancer cell growth inhibition. Appl Biol Chem 59: 739-746, 2016.

23. Yokoyama WM: Production of monoclonal antibodies. Curr Protoc Cytom 2006: Appendix 3:Appendix 3J, 2006.

24. Yokoyama WM, Christensen M, Santos GD and Miller D: Production of monoclonal antibodies. Curr Protoc Immunol 74: $1-25,2006$.

25. Kim D, Kwon S, Rhee JW, Kim KD, Kim YE, Park CS, Choi MJ, Suh JG, Kim DS, Lee Y, et al: Production of antibodies with peptide-CpG-DNA-liposome complex without carriers. BMC Immunol 12: 29, 2011

26. Kwon S, Kim YE, Park JA, Kim DS, Kwon HJ and Lee Y: Therapeutic effect of a TM4SF5-specific peptide vaccine against colon cancer in a mouse model. BMB Rep 47: 215-220, 2014. 